\title{
Pulmonary strongyloidiasis presenting micronodules on chest computed tomography
}

\author{
Kyungsoo Bae ${ }^{1,2}$, Kyung Nyeo Jeon ${ }^{1,2}$, Ji Young $\mathrm{Ha}^{2}$, Jong Sil Lee ${ }^{3}$, Byoung-Kuk Na ${ }^{4}$ \\ ${ }^{1}$ Department of Radiology, Institute of Health Sciences, Gyeongsang National University School of Medicine, Jinju, Korea; ${ }^{2}$ Department of \\ Radiology, Gyeongsang National University Changwon Hospital, Changwon, Korea; ${ }^{3}$ Department of Pathology, ${ }^{4}$ Department of Parasitology and \\ Tropical Medicine, and Institute of Health Sciences, Gyeongsang National University School of Medicine, Jinju, Korea \\ Correspondence to: Kyung Nyeo Jeon, MD, PhD. Department of Radiology, Gyeongsang National University School of Medicine, Jinju, Korea; \\ Department of Radiology, Gyeongsang National University Changwon Hospital, 555 Samjeongja-dong, Seongsan-gu, Changwon 51472, Korea. \\ Email: knjeon@gnu.ac.kr.
}

Submitted Mar 22, 2018. Accepted for publication Jul 03, 2018.

doi: $10.21037 /$ jtd.2018.07.32

View this article at: http://dx.doi.org/10.21037/jtd.2018.07.32

\section{Introduction}

Strongyloidiasis is chronic parasite infection caused by Strongyloides stercoralis. It is endemic in tropical and subtropical areas $(1,2)$. Skin contact with dirty soil and poor sanitary conditions are risk factors for infection. Rhabditiform larvae in warm and moist soil can develop into filariform larvae that can penetrate human skin and migrate through circulation to lungs (3). These larvae escape to alveolar space and migrate to pharynx. By being swallowed in pharyngeal secretion, larvae can settle in small intestine and mature into adults. If immune systems are compromised, the parasite is capable of autoinfection in the same host without further exogenous infestation (Figure 1) (3). Autoinfection can lead to massive and disseminated life-threatening parasitic infestation called hyperinfection syndrome. Multiple organs can be affected in hyperinfection syndrome. However, most presentations involve the lung and gastrointestinal tracts. In nonendemic area, strongyloidiasis often presents a diagnostic challenge (4). Here, we report a case of fatal pulmonary strongyloidiasis in a 65 -year-old male patient showing very uncommon findings on chest computed tomography (CT).

\section{Case presentation}

A 65-year-old Korean male visited the emergency room at a tertiary referral hospital. He was complaint of dyspnea, abdominal pain, and poor oral intake for 3 days. For several months, he had been intermittently taking corticosteroids
$(10-40 \mathrm{mg} / \mathrm{d})$ and non-steroidal anti-inflammatory drugs prescribed by a private clinic for back pain. Other past medical history was unremarkable. On admission, the patient was looking acutely ill with body temperature of $37.4^{\circ} \mathrm{C}$. Laboratory test revealed leukocytosis $\left(13,500 / \mathrm{mm}^{3}\right)$ and oxygen saturation measured using a pulse oximeter in room air was $89 \%$. Eosinophil count was within normal range $(0.6 \%)$. C-reactive protein level was increased (317 mg/L). Hemoglobin and serum albumin levels were low (9.7 and $2.8 \mathrm{~g} / \mathrm{dL}$, respectively).

Initial chest $\mathrm{X}$-ray was unremarkable. However, chest CT showed multiple micronodules ( $<3 \mathrm{~mm}$ in diameter) and faint ground-glass opacities in both lungs (Figure 2). Abdominal CT scan showed mild intrahepatic duct dilatation with normal extrahepatic ducts as seen in Clonorchiasis sinensis infestation. Serological test for $C$. sinensis was positive. Upper gastrointestinal endoscopy showed diffuse edematous gastric mucosal change and biopsy specimen showed chronic gastritis. Based on radiologic findings and test results, miliary tuberculosis or atypical pneumonia was suspected. Since sputum smear for acid-fast bacilli was negative, the patient was treated empirically with intravenous antibiotics (ceftazidime $2 \mathrm{~g}$ t.i.d, and ciprofloxacin $400 \mathrm{mg}$ b.i.d). Treatment of $C$. sinensis was also performed with praziquantel $(25 \mathrm{mg} / \mathrm{kg}$ t.i.d for 2 days).

However, patient's respiratory symptoms became worse. He expectorated blood-tinged sputum on the fifth day of admission. Follow-up chest X-ray showed bilateral ill- 


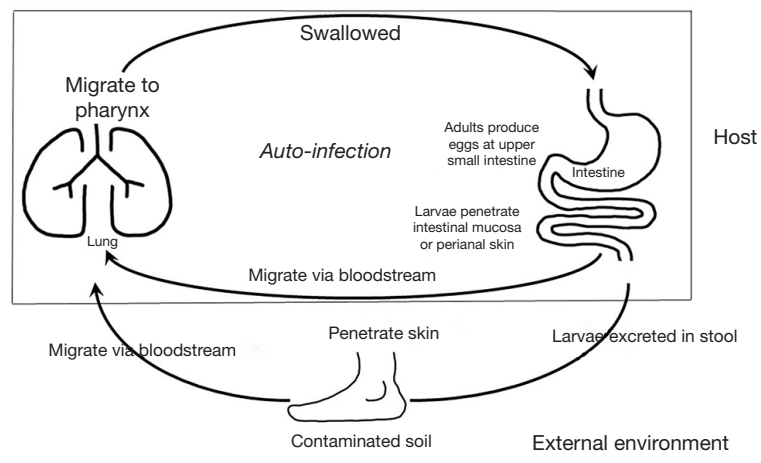

Figure 1 Life cycle of Strongyloides stercoralis in humans.

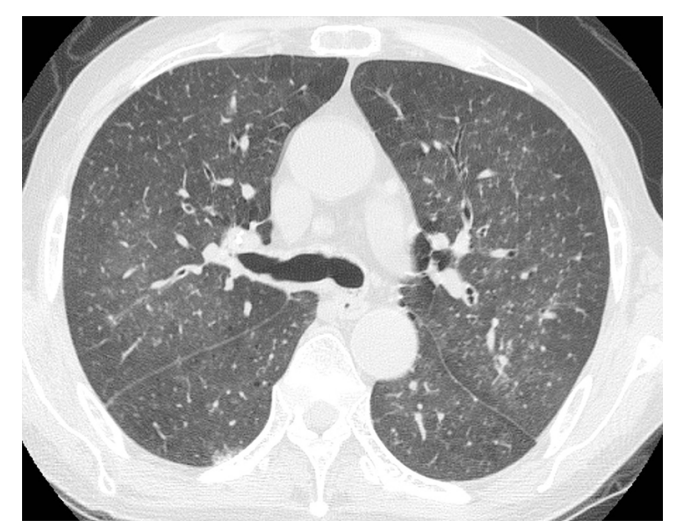

Figure 2 Initial chest CT image showing multiple micronodules $(<3 \mathrm{~mm})$ with predominant centrilobular distribution and faint ground-glass opacities in both lungs.

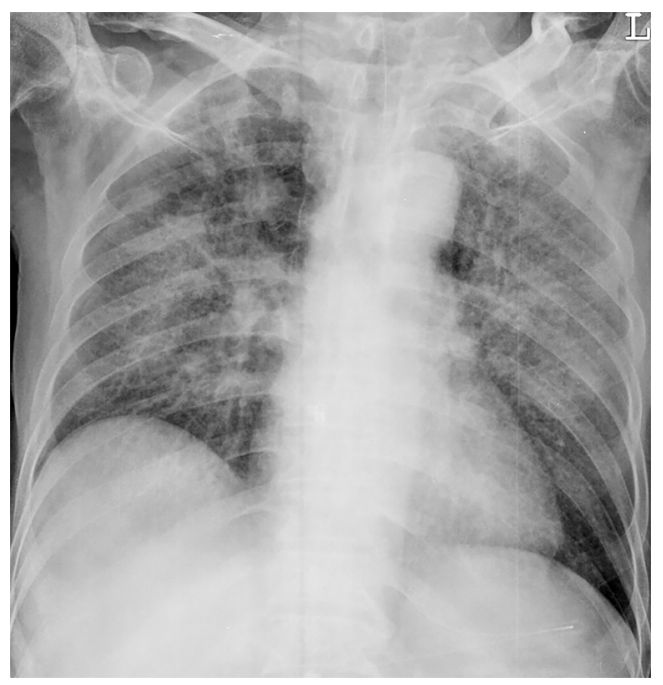

Figure 3 Follow-up chest X-ray on the 5 th day of admission showing bilateral ill-defined infiltrates with reticulonodular opacities.

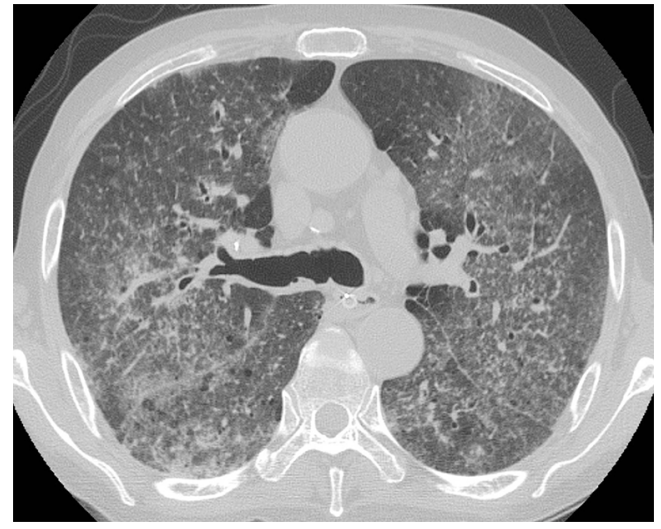

Figure 4 Follow-up chest CT showing increased number of micronodules and diffuse ground-glass opacities in both lungs.

defined infiltrates with reticulonodular opacities (Figure 3). Chest CT showed increased profusion of micronodules and diffuse ground-glass opacities (Figure 4). On bronchoscopic examination, there was no endobronchial lesion. However, bloody bronchoalveolar lavage (BAL) fluid was obtained, suggesting pulmonary alveolar hemorrhage. Smear of BAL fluid demonstrated third stage filariform larvae of Strongyloides stercoralis within clusters of red blood cells (Figures 5 and 6). The patient's wife disclosed that he stayed in China for several weeks 3 years ago. BAL findings for bacteria, fungus, and acid-fast bacilli were negative. The patient received anthelmintic treatment with albendazole $400 \mathrm{mg}$ b.i.d for 3 days. The same medication was repeated after 2 weeks. Patient's symptoms were temporarily improved after medication. However, he eventually progressed to respiratory failure due to superimposed Enterobacter cloacae pneumonia. He died 12 days later (on the 26th day after admission) despite intensive care.

\section{Discussion}

Strongyloides has a unique feature among human parasites in its capability of re-infecting their host without further exogenous infestation (1). This is called autoinfection (Figure 1). In the small intestine, some rhabditiform larvae hatched from eggs can metamorphose into infective filariform larvae. These filariform larvae can penetrate intestinal mucosa or perianal skin to establish a cycle of repeated endogenous infection. As such, S. stercoralis can infect a host for several years or even decades without detection after the host has left an endemic area (5). Immunocompetent hosts are commonly asymptomatic. 


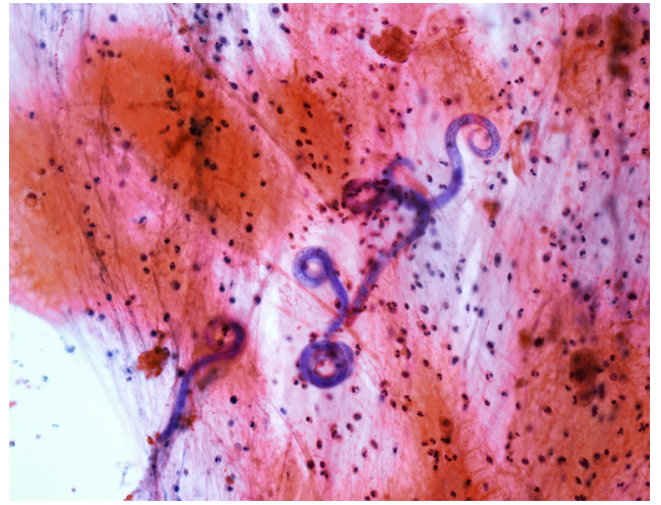

Figure 5 Microscopic examination of bronchoalveolar lavage fluid revealing the presence of Strongyloides stercoralis larvae in hemorrhagic background.

However, in immunocompromised hosts, the autoinfection cycle can result in overproduction of larvae in intestine and hematogenous dissemination into extraintestinal organs such as lungs and the central nervous system, leading to Strongyloides hyperinfection syndrome. Strongyloides hyperinfection can be fatal with mortality rate ranging from $50 \%$ to $80 \%$ (3). Secondary enteric bacterial infection is often the immediate cause of death. This was also the case for our patient. Disseminated Strongyloides with hyperinfection syndrome has been reported in patients with impaired cell-mediated immunity such as AIDS, diabetes mellitus, underlying malignancy, and in those treated with corticosteroids or cytotoxic drugs (6-8). Our patient might have been infected 3 years ago and sustained it through a low level of autoinfection. Recent administration of corticosteroids for his back pain might have triggered rapid production and dissemination of larvae.

We could not suspect $S$. stercoralis infection until larvae were found in BAL fluid. Although initial laboratory examinations showed leukocytosis, there was no peripheral blood eosinophilia. Peripheral blood eosinophilia can be absent in strongyloidiasis, particularly in cases with disseminated infection or hyperinfection. The reason of low eosinophil counts is unknown. Impaired immunity in patients with disseminated disease or hyperinfection might be a possible explanation (3). In our patient, stool examination failed to reveal S. stercoralis. Sensitivity of stool examination is low, particularly in non-endemic area (4). Therefore, examinations should be repeated over consecutive days if strongyloidiasis is suspected.

Cytology of bronchial specimen is a very reliable method

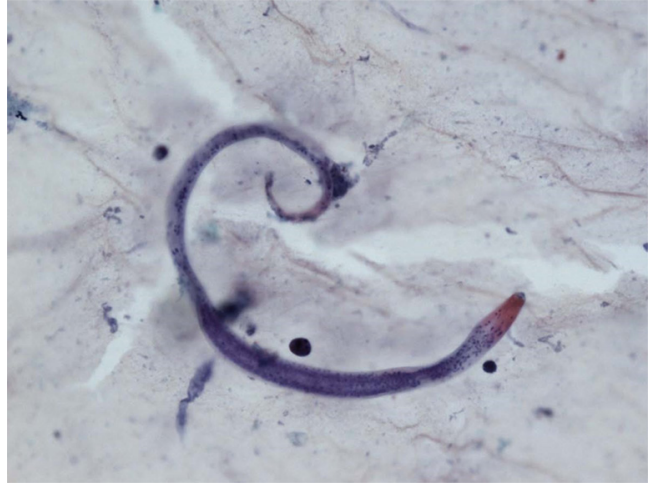

Figure 6 A Papanicolaou stained bronchoalveolar lavage smear showing a filariform larva of Strongyloides stercoralis $(\times 200)$.

to diagnose lung parasitosis (9). In the present case, correct diagnosis of $S$. stercoralis was made through cytological examination of BAL fluid. Preservation of larvae in the specimen was excellent and only simple staining technique such as Papanicolaou staining was required to disclose morphological features of the parasite.

In clinical setting, radiologic examinations are often on the front line in the detection and differential diagnosis of pulmonary diseases. According to previous studies, illdefined, patchy, and migratory infiltrates are common findings of pulmonary strongyloidiasis in chest $\mathrm{X}$-rays $(10,11)$. Ground-glass opacity and interlobular septal thickening are reported to be frequent findings in chest CT (12). Pulmonary consolidation, cavitation, and pleural effusion also occur (10). However, numerous micronodules on chest computed tomography shown in the present case have seldom received attention. In immunocompromised patients, this pattern can often be considered as urgent medical condition caused by lymphohematogenous dissemination of infectious organisms (13). A classic example is tuberculosis. CT scan of our patient showed numerous micronodules with centrilobular distribution and groundglass opacities. In the present case, micronodules aggravated in follow-up represented disseminated massive $S$. stercoralis larvae which impacted pulmonary circulation supplying centrilobular core (14). When these larvae migrated from the capillary bed into the alveoli, hemorrhage and inflammation occurred in the alveolar space and interstitium which manifested as superimposed ground-glass opacities. Retrospective analysis of CT findings clearly explained this pathogenesis of the disease. 
Among treatment options for strongyloidiasis including thiabendazole, albendazole, and ivermectin, ivermectin has become the gold standard of care. It has been proven to be more effective and better tolerated than thiabendazole or albendazole $(15,16)$. However, albendazole is still used in some countries due to low availability of ivermectin, as seen in our case (16).

In conclusion, with increased globalization, strongyloidiasis can occur in non-endemic area. However, diagnosis might be challenging due to non-specific clinical features. Thus, a high level of suspicion is mandatory for early diagnosis. In immunocompromised patients with history of residence in or visit to endemic areas, pulmonary strongyloidiasis should be included in differential diagnosis of numerous micronodules on chest radiologic examinations.

\section{Acknowledgements}

We thank Keunyoung Lina Bae for the English-language review.

\section{Footnote}

Conflicts of Interest: The authors have no conflicts of interest to declare.

Informed Consent: The requirement for written informed consent for publishing this report and any accompanying images was waived by the Institutional Review Board (GNUCH 2018-03-009).

\section{References}

1. Schär F, Trostdorf U, Giardina F, et al. Strongyloides stercoralis: Global Distribution and Risk Factors. PLoS Negl Trop Dis 2013;7:e2288.

2. Ngui R, Halim NA, Rajoo Y, et al. Epidemiological Characteristics of Strongyloidiasis in Inhabitants of Indigenous Communities in Borneo Island, Malaysia. Korean J Parasitol 2016;54:673-8.

3. Lam CS, Tong MK, Chan KM, et al. Disseminated strongyloidiasis: a retrospective study of clinical course and outcome. Eur J Clin Microbiol Infect Dis 2006;25:14-8.

4. Sato Y, Kobayashi J, Toma H, et al. Efficacy of stool examination for detection of Strongyloides infection. Am J Trop Med Hyg 1995;53:248-50.
5. Gill GV, Beeching NJ, Khoo S, et al. A British Second World War veteran with disseminated strongyloidiasis. Trans R Soc Trop Med Hyg 2004;98:382-6.

6. Lessnau KD, Can S, Talavera W. Disseminated Strongyloides stercoralis in human immunodeficiency virus-infected patients. Treatment failure and a review of the literature. Chest 1993;104:119-22.

7. Won EJ, Jeon J, Koh YI, et al. Strongyloidiasis in a diabetic patient accompanied by gastrointestinal stromal tumor: cause of eosinophilia unresponsive to steroid therapy. Korean J Parasitol 2015;53:223-6.

8. Kia EB, Rahimi HR, Mirhendi H, et al. A case of fatal strongyloidiasis in a patient with chronic lymphocytic leukemia and molecular characterization of the isolate. Korean J Parasitol 2008;46:261-3.

9. Grapsa D, Petrakakou E, Botsoli-Stergiou E, et al. Strongyloides stercoralis in a bronchial washing specimen processed as conventional and Thin-Prep smears: report of a case and a review of the literature. Diagn Cytopathol 2009;37:903-5.

10. Kothary NN, Muskie JM, et al. Strongyloides stercoralis hyperinfection. Radiographics 1999;19:1077-81.

11. Woodring JH, Halfhill H 2nd, Berger R, et al. Clinical and imaging features of pulmonary strongyloidiasis. South Med J 1996;89:10-9.

12. Nabeya D, Haranaga S, Parrott GL, et al. Pulmonary strongyloidiasis: assessment between manifestation and radiological findings in 16 severe strongyloidiasis cases. BMC Infect Dis 2017;17:320.

13. Walker CM, Abbott GF, Greene RE, et al. Imaging pulmonary infection: classic signs and patterns. AJR Am J Roentgenol 2014;202:479-92.

14. Webb WR. Thin-section CT of the secondary pulmonary lobule: anatomy and the image--the 2004 Fleischner lecture. Radiology 2006;239:322-38.

15. Segarra-Newnham M. Manifestations, diagnosis, and treatment of Strongyloides stercoralis infection. Ann Pharmacother 2007;41:1992-2001.

16. Buonfrate D, Requena-Mendez A, Angheben A, et al. Severe strongyloidiasis: a systematic review of case reports. BMC Infect Dis 2013;13:78.

Cite this article as: Bae K, Jeon KN, Ha JY, Lee JS, Na BK. Pulmonary strongyloidiasis presenting micronodules on chest computed tomography. J Thorac Dis 2018;10(8):E612-E615. doi: 10.21037/jtd.2018.07.32 